\title{
Irreducible inguinal hernia with intrasaccular perforated sigmoid malignancy
}

\author{
Pari Muthukumar, Apoorva Prathap, Ezhilan Subramanian, Vishwanath Pai \\ Department of General Surgery, Sri Ramachandra Medical College and Hospital, India
}

Received: October 11, 2015

Accepted: December 31, 2015 Online Published: March 10, 2016

DOI: $10.5430 /$ css.v2n2p27

URL: http://dx.doi.org/10.5430/css.v2n2p27

\begin{abstract}
Irreducible inguinal hernias are commonly encountered in an emergency setting. The content is usually small bowel or omentum and diagnosis is made by physical examination. We present a case of an irreducible inguinal hernia, which on opening of the sac revealed a perforated sigmoid colonic growth as the content.
\end{abstract}

Key Words: Irreducible inguinal hernia, Sigmoid adenocarcinoma, Intrasaccularperforated malignancy, Uncommon hernial content

\section{INTRODUCTION}

About $75 \%$ of hernias occur in the groin ${ }^{[1]}$ and about $96 \%$ of groin hernias are inguinal. ${ }^{[2]}$ Inguinal hernia repair is one of the most commonly performed surgeries. In spite of this, hernia repair poses to be a challenge to surgeons due to the wide variety of content one can expect to find in the sac. Malignant tumours as content are relatively rare and found in approximately $0.5 \%$ of hernias. ${ }^{[3]}$ Literature shows that only a handful of these tumours were found to be perforated on opening of the sac. They can involve but are not limited to the small intestine, sigmoid colon or caecum. The incidence of colorectal carcinoma increases with age, with about $90 \%$ of new cases occurring in individuals above the age of 50. ${ }^{[4]}$ We present a case of an irreducible inguinal hernia containing a perforated sigmoid carcinoma.

\section{CASe presentation}

A 37-year-old gentleman presented to our Emergency Department with complaints of abdominal pain and vomiting for a week. He gave a history of a large mass in the region of the left groin, which had been present for the past 10 years.

On physical examination, he was found to be afebrile with tachycardia. His abdomen was mildly distended with sluggish bowel sounds. A large, tender, irreducible indirect hernia was present in his left inguinal region. Content appeared to be bowel.

In view of impending strangulation, patient was taken up for emergency surgery. The mass was approached through a left inguinoscrotal incision (see Figure 1). Opening of the sac revealed the content to be a perforated sigmoid colon (see Figure 2). The surgery was converted to a laparotomy and a Hartman's procedure was performed. Hernia repair was achieved through herniorrhaphy by Darning method.

The post-operative course was uneventful.Histopathology report revealed adenocarcinoma of the sigmoid colon with no nodal involvement. Margins were free of tumour. Colonoscopy through the colostomy was done to rule out

\footnotetext{
*Correspondence: Ezhilan Subramanian; Email: drezhilan@gmail.com; Address: Department of General Surgery, Sri Ramachandra Medical College and Hospital, India. 
synchronous lesions. A CT abdomen ruled out secondaries. After 6 cycles of chemotherapy, he underwent laparoscopic reversal of the Hartman's procedure.

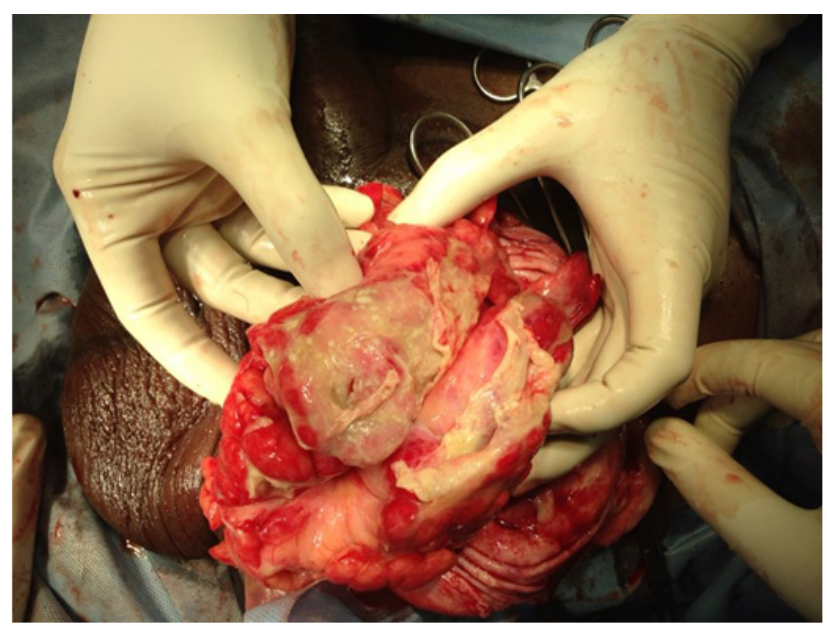

Figure 1. Irreducible inguinal hernia delivered from the scrotum

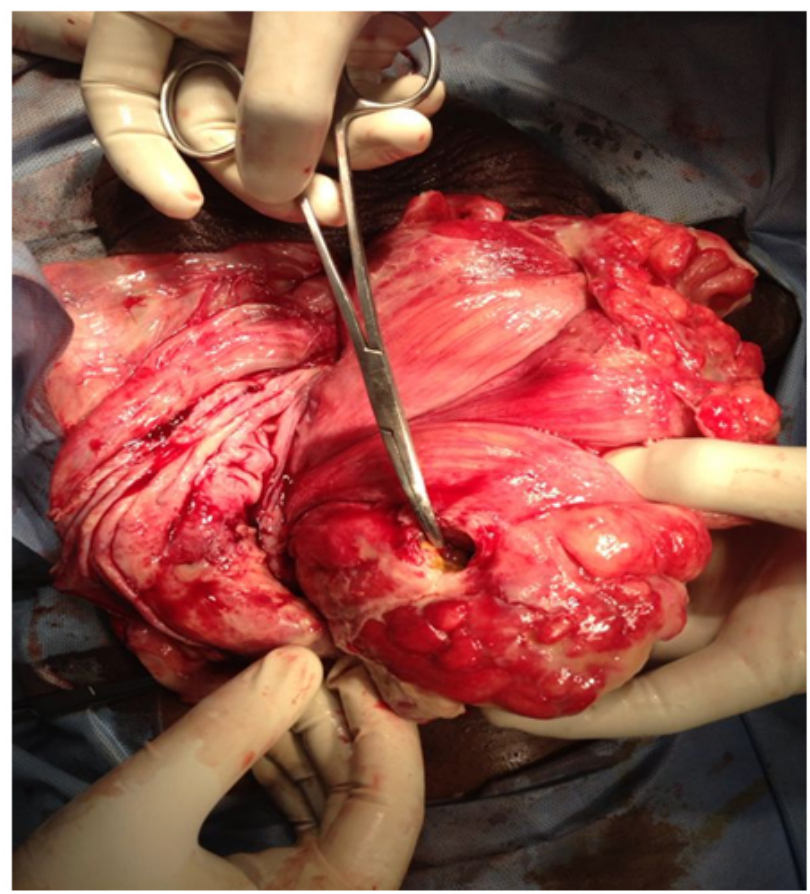

Figure 2. Perforation noted in the sigmoid colon

\section{Discussion}

With an incidence of less than $0.5 \%$, malignancies in inguinal hernias are unusual. ${ }^{[5]}$ Based on their relationship to the hernial sac, intra-abdominal malignancies can be classified as saccular or intra-saccular. ${ }^{[6]}$ Saccular tumours are primary tumours arising from the peritoneal surface of the sac and intra-saccular tumours are tumours arising from the intra-abdominal organs contained in the sac. While inguinal hernias and colorectal carcinoma are common findings individually, the occurrence of colorectal carcinoma as an intrasaccular finding is rare. ${ }^{[7]}$

A review of literature shows us that there are less than 5 reported cases of a perforated sigmoid carcinoma found in an incarcerated inguinal hernia. Such cases are associated with abscess formation and generalized peritonitis. ${ }^{[8]}$ But in our patient, the perforated intrasaccular malignancy turned out to be a blessing in disguise as the hernia sac served to contain the perforation, thus limiting the sequelae of peritonitis.

Another point to note is the presence of sigmoid adenocarcinoma in our relatively young patient. Since colorectal carcinoma is not common in age groups below 50, we did not consider the possibility of an intrasaccular malignancy. The diagnosis of an inguinal hernia is a clinical one. Ultrasound is not useful in cases of complicated hernia; ${ }^{[8]}$ in such instances a CT scan is recommended.

Literature tells us that most of the intrasaccular tumours were found to be sigmoid adenocarcinomas, discovered as an incidental finding on table. ${ }^{[8]}$ The incidence of colorectal carcinoma in longstanding inguinal hernias prompts us to screen patients with such hernias with a flexible sigmoidoscopy, colonoscopy ${ }^{[5]}$ or a CT scan.

\section{Conclusion}

Findings of intrasaccular tumours are not common and should be thought of when a longstanding hernia turns irreducible. Our case shows that in spite of the fact that colorectal carcinoma is rare in the age group below 40; a screening CT must be advocated in patients with a large, long standing left sided inguinal hernia.

\section{CONFlicts of InTEREST Disclosure}

The authors declare that they have no conflicts of interest.

\section{REFERENCES}

[1] Schwartz's Principles of Surgery. 9th edition. Chapter 35 - Abdominal Wall, Omentum, Mesentery and Retroperitoneum. 2439-2443 p.
[2] Available from: http://www.uptodate.com/contents/colo rectal-cancer-epidemiology-risk-factors-and-prote ctive-factors\#H1

[3] Bali C, Tsironis A, Zikos N, et al. An unusual case of a stran- 
gulated right inguinal hernia containing the sigmoid colon. International Journal of Surgery Case Reports. 2011; 2(4): 53-55. http://dx.doi.org/10.1016/j.ijscr.2011.01.005

[4] Available From: http://www.cancer.org/cancer/colonand rectumcancer/

[5] Tan GYM, Guy RJ, Eu KW. Obstructing sigmoid cancer with local invasion in an incarcerated inguinal hernia. ANZ Journal of Surgery. 2003; 73(1-2): 80-82. http://dx.doi.org/10.1046/j.1445-2 $197.2003 .02623 . \mathrm{x}$

[6] Meniconi RL, Vennarecci G, Lepiane P, et al. Locally advanced carci- noma of the cecum presenting as a right inguinal hernia: a case report and review of the literature. Journal of Medical Case Reports. 2013; 7(1): 206. http://dx.doi.org/10.1186/1752-1947-7-206

[7] Mandava N, Kumar S, Pizzi WF, et al. Perforated colorectal carcinomas. The American Journal of Surgery. 1996; 172(3): 236-238. http://dx.doi.org/10.1016/S0002-9610(96)00164-X

[8] Ko KH, Yu CY, Kao CC, et al. Perforated sigmoid colon cancer within an irreducible inguinal hernia: a case report. Korean Journal of Radiology. 2010; 11(2): 231-3. http://dx.doi .org/10.3348 /kjr.2010.11.2.231 